\title{
On the pure Jacobi sums
}

\author{
by \\ Shigeki Akiyama (Niigata)
}

Let $p$ be an odd prime and $\mathbb{F}_{q}$ be the field of $q=p^{2}$ elements. We consider the Jacobi sum over $\mathbb{F}_{q}$ :

$$
J(\chi, \psi)=\sum_{x \in \mathbb{F}_{q}} \chi(x) \psi(1-x),
$$

where $\chi$ and $\psi$ are non-trivial characters of $\mathbb{F}_{q}^{\times}$, whose value at 0 is defined to be 0 . It is well known that the absolute value of $J(\chi, \psi)$ is $\sqrt{q}=p$ whenever $\chi \psi$ is not principal. Following [9], [11], call the Jacobi sum $J(\chi, \psi)$ pure if $J(\chi, \psi) / p$ is a root of unity.

Let $\operatorname{ord}(\chi)$ be the order of $\chi$ in $\widehat{\mathbb{F}_{q}^{\times}}$. We assume that $\operatorname{ord}(\psi)=2$ and $\operatorname{ord}(\chi)=n \geq 3$. This special type of Jacobi sums plays an important role in evaluating the argument of the Gauss sum

$$
G(\chi)=\sum_{x \in \mathbb{F}_{q}} \chi(x) \zeta_{p}^{\operatorname{Tr}_{\mathbb{F}_{q} / \mathbb{F}_{p}}(x)},
$$

where $\zeta_{p}$ is a primitive $p$ th root of unity (see [2], [3]). Moreover, recently the rationality of this Jacobi sum is used to characterize the irreducible module of the Terwilliger algebras of cyclotomic association schemes (see [10]).

In this note, we prove

THEOREM. $J(\chi, \psi)$ is pure if and only if one of the following four conditions holds:

1. $n$ is a divisor of $p+1$,

2. $n=2(p-1) / k$ with an odd integer $k$,

3. $n=24$ and $p \equiv 17,19(\bmod 24)$,

4. $n=60$ and $p \equiv 41,49(\bmod 60)$.

Further, $J(\chi, \psi)= \pm p$ in all four cases.

There are numerous results concerning the determination of Gauss and Jacobi sums. See e.g. [2], [3], [8]. A nice historical survey is found in [4]. The same type "purity" problems for the case of Gauss sums are treated in [6], 
[13], [1], [7], [9], [11]. But it seems that no such concrete result on Jacobi sums is known. Although our essential tool is the theorem of Stickelberger, the argument for the "only if" part is elementary and rather complicated. The author feels somewhat curious that this simple result is derived by such a brute force method.

We first see

Proposition. Let $S_{1}=\left\{x \in(\mathbb{Z} / n \mathbb{Z})^{\times}: x \equiv i(\bmod n)\right.$ for $i \in[1, n / 2) \cap$ $\mathbb{Z}\}$, and $S_{2}=(\mathbb{Z} / n \mathbb{Z})^{\times} \backslash S_{1}$. Then $J(\chi, \psi)$ is pure if and only if there exists $x \in S_{1}$ such that $x S_{1}=S_{1}$ and $p \equiv-x(\bmod n)$.

Pr o of. By the Theorem of Hasse-Davenport (see Theorem 5.1 of [12]) and the well known result on the sign determination of Gauss sums of order 2 for the prime field $\mathbb{F}_{p}$, we get $G(\psi)=(-1)^{(p+1) / 2} p$. The theorem of Stickelberger (see Theorem 2.2 of [12]) states that $J(\chi, \psi) / p= \pm G(\chi) / G(\chi \psi)$ is a unit of the integer ring of $\mathbb{Q}\left(\zeta_{n}\right)$ if and only if

$$
\left\{\frac{a}{n}\right\}+\left\{\frac{p a}{n}\right\}=\left\{\frac{a}{n}+\frac{1}{2}\right\}+\left\{p\left(\frac{a}{n}+\frac{1}{2}\right)\right\} \quad \text { for all } a \text { with }(a, n)=1 .
$$

Here $\{x\}=x-[x]$ and $[x]$ is the greatest integer not exceeding $x$. As $p$ is odd, we have to check the conditions for $a \in S_{1}$ only, because the condition is symmetric with respect to $a \leftrightarrow n-a$. Thus we choose $a$ with $1 \leq a \leq n / 2$ and $(a, n)=1$. Then

$$
\frac{a}{n}+\left\{\frac{p a}{n}\right\}=\frac{a}{n}+\frac{1}{2}+\left\{\frac{p a}{n}+\frac{1}{2}\right\} \quad \text { for } a \in S_{1} .
$$

Note that the condition depends only on $p(\bmod n)$. We see that $(1)$ is equivalent to $\{p a / n\} \in[1 / 2,1)$ for $a \in S_{1}$. Put $p=n y-x$ with integers $x, y$. Then $x(\bmod n) \in S_{1}$ and $\{x a / n\}$ must lie in the interval $[0,1 / 2)$. Noting that $|J(\chi, \psi)|=p$ and $\mathbb{Q}\left(\zeta_{n}\right) / \mathbb{Q}$ is an abelian extention, $J(\chi, \psi) / p$ is a root of unity under these conditions. This shows the assertion.

Re m a r k. Consider the Jacobi sum on the general finite field $\mathbb{F}_{q}$ with $q=$ $p^{f}$, for a while. Then, similarly to the above proof, we can easily show that, if the extension degree $f$ is odd, then there are no $\chi$ for which $J(\chi, \psi) / \sqrt{q}$ is a root of unity. Our Theorem concerns the first non-trivial case.

The sufficiency of the conditions of the Theorem follows immediately since:

- $1 \times S_{1}=S_{1} \leftrightarrow$ Condition 1 .

- $n \equiv 0(\bmod 4)$ and $(n / 2-1) S_{1}=S_{1} \leftrightarrow$ Condition 2 .

- If $n=24$ and $S_{1}=\{\widetilde{1}, \widetilde{5}, \widetilde{7}, \widetilde{11}\}$, then $\widetilde{5} S_{1}=S_{1}$ and $\widetilde{7} S_{1}=S_{1} \leftrightarrow$ Condition 3. 
- If $n=60$ and $S_{1}=\{\widetilde{1}, \widetilde{7}, \widetilde{11}, \widetilde{13}, \widetilde{17}, \widetilde{19}, \widetilde{23}, \widetilde{29}\}$, then $\widetilde{11} S_{1}=S_{1}$ and $\widetilde{19} S_{1}=S_{1} \leftrightarrow$ Condition 4 .

Here we denote by $\widetilde{x}$ the $\operatorname{coset} x(\bmod n)$. Our next task is to show that $J(\chi, \psi)$ is real in the above four cases. The first two cases are handled easily.

LEMma 1. We have $J(\chi, \psi)= \pm p$ whenever $n$ is a divisor of $p+1$ or $n=2(p-1) / k$ with an odd integer $k$.

Proof. We have

$$
J(\chi, \psi)=\sum_{x \in \mathbb{F}_{q}} \chi\left(x^{p}\right) \psi\left(1-x^{p}\right)=\sum_{x \in \mathbb{F}_{q}} \chi^{p}(x) \psi^{p}(1-x)=J\left(\chi^{p}, \psi\right) .
$$

If $p \equiv-1(\bmod n)$ then

$$
J\left(\chi^{p}, \psi\right)=J(\bar{\chi}, \psi)=\overline{J(\chi, \psi)} .
$$

This shows the first case. For the second case, we have $p-1 \equiv n k / 2 \equiv n / 2$ $(\bmod n)$ and $\chi^{p-1}=\chi^{n / 2}=\psi$, as $n=\operatorname{ord}(\chi)$. By using $(2)$ and $\psi(-1)=1$, we have

$$
\begin{aligned}
J(\chi, \psi) & =\sum_{x \in \mathbb{F}_{q}} \chi^{p}(x) \psi(1-x)=\sum_{x \in \mathbb{F}_{q}} \chi(x) \psi(x(1-x)) \\
& =\sum_{x \in \mathbb{F}_{q}^{\times}} \overline{\chi(x)} \psi\left(x^{-1}\left(1-x^{-1}\right)\right)=\sum_{x \in \mathbb{F}_{q}} \overline{\chi(x)} \psi(x-1) \\
& =\overline{J(\chi, \psi)} .
\end{aligned}
$$

This shows the assertion.

The case $n=24$ was already proved in [3]. We show this directly for the convenience of the reader.

Lemma 2. We have $J(\chi, \psi)= \pm p$ whenever $n=24$ and $p \equiv 17,19$ $(\bmod 24)$.

Proof. We have already shown that $J(\chi, \psi) / p$ is a root of unity of the field $\mathbb{Q}\left(\zeta_{24}\right)$. Let $\sigma_{k}$ be the element of $\operatorname{Gal}\left(\mathbb{Q}\left(\zeta_{24}\right) / \mathbb{Q}\right)$ with $\sigma_{k}\left(\zeta_{24}\right)=\zeta_{24}^{k}$. Then

$$
\sigma_{11}(J(\chi, \psi))=\sigma_{11}\left(G(\chi) G(\psi) / G\left(\chi^{13}\right)\right)=G\left(\overline{\chi^{13}}\right) G(\psi) / G(\bar{\chi})=J(\chi, \psi) .
$$

This shows that $J(\chi, \psi) / p$, the 24 -th root of unity, is invariant under $\sigma_{11}$, proving the assertion.

The first manuscript of this note did not contain the following proof of Lemma 3, which is essentially due to Mieko Yamada. The author tried in vain to do this. She also informed me that a more general assertion concerning Lemmas $1-3$ is presented in [10] and the detailed version of it will contain its proof. 
Lemma 3. We have $J(\chi, \psi)= \pm p$ whenever $n=60$ and $p \equiv 41,49$ $(\bmod 60)$.

Proof. As in Lemma 2 , we see $\sigma_{29}(J(\chi, \psi))=(J(\chi, \psi))$. Thus $J(\chi, \psi) \in$ $\mathbb{Q}(\sqrt{-1})$ and $J(\chi, \psi) / p$ is equal to \pm 1 or $\pm \sqrt{-1}$. Note that

$$
(J(\chi, \psi))^{5} \equiv J\left(\chi^{5}, \psi\right)(\bmod 5) .
$$

As $\operatorname{ord}\left(\chi^{5}\right)=12$, we see that there exist rational integers $C, D$ with $p=C^{2}+D^{2}$ and

$$
J\left(\chi^{5}, \psi\right)=-(C+D \sqrt{-1})^{2},
$$

by Theorems 4.8 and 4.10 of [3]. We easily see that $C D \equiv 0(\bmod 5)$ as $p \equiv \pm 1(\bmod 5)$. This shows the assertion.

$\mathrm{R}$ e $\mathrm{m}$ a r $\mathrm{k}$. There is a remaining problem to determine the sign of $J(\chi, \psi)$ when it is real. See [3] for the first three cases of the Theorem. If $n=60$ and $p \equiv 41,49(\bmod 60)$, the congruence relation $(3)$ and Theorems 4.8 and 4.10 of [3] are enough to determine the sign ambiguity of $J(\chi, \psi)$. Summing up, we have

$$
J(\chi, \psi)= \begin{cases}p, & \text { for cases } 1 \text { and } 3 \\ (-1)^{(p+1) / 2} p, & \text { for case } 2 \\ \pm\left(\frac{p}{3}\right) p, & \text { for case } 4\end{cases}
$$

Here \pm of the last case is $+($ resp. -$)$ when $A \equiv 0(\bmod 5)($ resp. $\not \equiv)$, with a positive odd integer $A$, which is uniquely determined by $p=A^{2}+B^{2}$.

Now we show the necessity of the conditions of the Theorem. For convenience, we identify the element of $S_{1}\left(\right.$ resp. $\left.S_{2}\right)$ with the integer in $[1, n / 2)$ (resp. $[n / 2, n)$ ) in the later proof.

Lemma 4. Besides case 1 of the Theorem, if $J(\chi, \psi)$ is pure then $n=$ $\operatorname{ord}(\chi)$ is divisible by 4 .

Proof. Assume $n$ is odd and $a \neq 1$ is an integer with $a S_{1}=S_{1}$. Choose an integer $i \geq 1$ such that $n / 2^{i+1}<a \leq n / 2^{i}$. Then $a \neq 1$ implies $1 \leq$ $n / 2^{i+1}$. Thus $2^{i} \in S_{1}$ and $2^{i} a \in S_{2}$, which contradicts $a S_{1}=S_{1}$. Thus by the Proposition, $n$ must be even. Now if $n=2 m$ and $m$ is odd, then similarly we choose $a \in S_{1}$ and an integer $i$. Noting that $a$ is odd, we have

$$
a\left(\frac{n}{2}-2^{i}\right) \equiv \frac{n}{2}-2^{i} a(\bmod n) .
$$

We get $n / 2-2^{i} \in S_{1}$ and $n / 2-2^{i} a \in S_{2}$, which contradicts $a S_{1}=S_{1}$.

The next step is a prototype of our following arguments, which seems somewhat curious at first glance.

Lemma 5. Besides cases 1 or 2 of the Theorem, if $n$ is divisible by 8 and $n>14^{2}$, then $J(\chi, \psi)$ is not pure. 
Pr o o f. Let

$$
T(a, b)=\left\{x \in(\mathbb{Z} / n \mathbb{Z})^{\times}: x \equiv i(\bmod n) \text { for } i \in[a, b) \cap \mathbb{Z}\right\}
$$

and define

$$
T_{i}=T\left(\frac{(i-1) n}{4}, \frac{i n}{4}\right) \quad(i=1,2,3,4) .
$$

We identify each element of $T_{i}$ with an integer in $[(i-1) n / 4$, in/4). Let $n=2^{e} m(e \geq 3)$ with $m$ odd. Consider a vector

$$
\begin{array}{r}
(A, B, C, D)=\left(\frac{n}{2^{e}}+2^{i}, \frac{n}{2^{e}}+2^{i+1}, \frac{n}{2^{e}}+\frac{n}{4}+2^{i}, \frac{n}{2^{e}}+\frac{n}{4}+2^{i+1}\right) \\
\text { for } i \geq 1 .
\end{array}
$$

Assume that $2^{i+4} \leq n$, which implies $A, B, C, D \in S_{1}$. As $n / 2$ is even, the condition $a S_{1}=S_{1}$ is equivalent to $(n / 2-a) S_{1}=S_{1}$ and $n / 2-a$ also lies in $S_{1}$. Thus if $J(\chi, \psi)$ is pure, we may assume $a \in[1, n / 4)$ and $a S_{1}=S_{1}$. We first treat the case $a \in[8, n / 4)$. Let $i \geq 1$ be an integer such that $a \in\left[n / 2^{i+2}, n / 2^{i+1}\right)$. (We can choose $i$ which satisfies $2^{i+4} \leq n$ in this case.) Then we have $a A \in T_{1}$ and $a B \in T_{2}$. In fact, $B-A=2^{i}$ and $2^{i} a(\bmod n)$ has a representative in $[n / 4, n / 2) \cap \mathbb{Z}$. Thus if $a A\left(\in S_{1}\right)$ lies in $T_{2}$ then $a B$ must be in $S_{2}$. Noting $a$ is odd, we consider two cases:

1. If $a \equiv 1(\bmod 4)$ then

$$
a D \equiv n / 4+a B(\bmod n),
$$

which implies $a D \in T_{3}$.

2. If $a \equiv 3(\bmod 4)$ then

$$
a C \equiv 3 n / 4+a A(\bmod n),
$$

which implies $a C \in T_{4}$.

Therefore when $a \in[8, n / 4)$, we have shown that, contrary to the assumption, $a A, a B, a C$ and $a D$ cannot lie in $S_{1}$ simultaneously.

There remains the case $a \in[1,7]$. The value 1 corresponds to case 1 or 2 of the Theorem. (Remember that we used the reflection $a \leftrightarrow n / 2-a$.) Thus the only remaining case is $a=3,5$ and 7 . But this case is easily handled because if $a S_{1}=S_{1}$ then $a^{2} S_{1}=S_{1}$ and $n / 4>7^{2}$ implies $a^{2} \in[8, n / 4)$.

Lemma 6. Besides cases 1 or 2 of the Theorem, if $n>10^{2}$ and $n=4 m$ and $(m, 3)=1$ then $J(\chi, \psi)$ is not pure.

Pr o of. In this case, consider a vector

$$
(A, B, C)=\left(\frac{n}{4}+2^{i}, \frac{n}{4}+2^{i+1}, \frac{n}{4}+3 \cdot 2^{i}\right) \quad \text { for } i \geq 1 .
$$

As $A, B$ and $C$ must be in $S_{1}$, we assume $3 \cdot 2^{i+2} \leq n$. Using the reflection $a \leftrightarrow n / 2-a$, we may choose $a \in[1, n / 4)$. First we assume that 
$a \in[6, n / 4)$ and $a S_{1}=S_{1}$. Choose the integer $i$ with $a \in\left[n / 2^{i+2}, n / 2^{i+1}\right)$. Then by the same argument as in Lemma 5 , we have $a A \in T_{1}$ and $a B \in T_{2}$. Thus $a C \in S_{2}$, which is a contradiction. The case $a=3$ and 5 is handled similarly.

In the following, we proceed similarly. In other words, we first choose four elements in $S_{1}$. Then we show that the $a$-multiple of these elements cannot lie in $S_{1}$ simultaneously. The later arguments become a little bit complicated, especially in Lemma 8.

Lemma 7. Besides cases 1 or 2 of the Theorem, if $n>46^{2}$ and $n=4 m$ and $m$ is odd and not square free, then $J(\chi, \psi)$ is not pure.

Proof. Let $q$ be an odd prime and $m$ is divisible by $q^{2}$. In this case, we take a vector

$(A, B, C, D)=\left(\frac{n}{4 q}+2^{i}, \frac{n}{4 q}+2^{i+1}, \frac{n}{4 q}+\frac{k n}{2 q}+2^{i}, \frac{n}{4 q}+\frac{k n}{2 q}+2^{i+1}\right) \quad$ for $i \geq 1$.

Here $k$ is a positive integer smaller than $3 q / 4$, which is taken suitably later. Assume that $3 \cdot 2^{i+4} \leq n$. Then

$$
\frac{n}{4 q}+\frac{k n}{2 q}+2^{i+1} \leq \frac{n}{12}+\frac{3 n}{8}+2^{i+1} \leq \frac{n}{2} .
$$

Thus $A, B, C$ and $D$ are contained in $S_{1}$. We first prove the case $a \in$ $[24, n / 4)$. Choose $k$ so that

$$
\frac{1}{4} \leq\left\{\frac{a k}{2 q}\right\} \leq \frac{3}{4} .
$$

This is possible. In fact, let $l \in[q / 2,3 q / 2) \cap \mathbb{Z}$ and solve the congruence for $x$ :

$$
a x \equiv l(\bmod 2 q) \quad \text { and } \quad x \in[1,2 q] \cap \mathbb{Z} .
$$

Define

$$
k(l)= \begin{cases}x & \text { for } x \leq q \\ 2 q-x & \text { for } x>q .\end{cases}
$$

Then (4) is satisfied for $k=k(l)$. It is easily shown that the number of distinct $k(l)$ is $(q+1) / 2$. So $k=k(l)$ can be taken smaller than, say, $3 q / 4$. Noting $3 \cdot 2^{i+4} \leq n$ and $a \geq 24$, we can choose an integer $i$ satisfying $a \in\left[n / 2^{i+2}, n / 2^{i+1}\right)$. Then, similarly to Lemma 5 , we can easily check that $a A \in T_{1}$ and $a B \in T_{2}$. By (4), we have $a k n /(2 q) \in T_{2} \cup T_{3}$. We consider two cases. If $a k n /(2 q) \in T_{2}$ then $a D \in S_{2}$. And if $a k n /(2 q) \in T_{3}$ then $a C \in S_{2}$. This completes the proof for $a \in[24, n / 4)$. Finally, we treat the remaining case $a \leq 23$. If $23 \geq a \geq 5$, then $a^{2} S_{1}=S_{1}$ and $24<a^{2}<n / 4$; these cases can be proved similarly. If $a=3$ then consider $27 S_{1}=S_{1}$. 
Lemma 8. Besides cases 1 or 2 of the Theorem, if $n>70^{2}$ and $n=12 m$ and $(m, 6)=1$ and $m$ has a prime factor greater than 6 , then $J(\chi, \psi)$ is not pure.

Proof. Let $q$ be the greatest prime factor of $q$. We assume $(n / q, q)=1$, in light of Lemma 7. In this case, we take a vector

$$
(A, B, C, D)=\left(\frac{n}{12} \pm 2^{i+1}, \frac{n}{12} \pm 3 \cdot 2^{i}, A+\frac{k n}{2 q}, B+\frac{k n}{2 q}\right) \quad \text { for } i \geq 1 .
$$

The signs in $A$ and $B$ are defined by

$$
n / 12 \pm 2^{i+1} \not \equiv 0(\bmod 3) \text {. }
$$

Here $k$ is a positive integer smaller than $3 q / 4$, which is chosen later. Assume that $3^{2} \cdot 2^{i+3} \leq n$. Then we see that $A, B, C$ and $D$ are in $[1, n / 2)$. Moreover, $A, B \in S_{1}$ and $C, D$ are coprime with 6 . But, in this case, $C, D$ may be divisible by $q$. Let $l \in[q / 2,3 q / 2) \cap \mathbb{Z}$ and $k(l)$ be the integer defined by (5) in Lemma 7 . Then there exist $(q+1) / 2$ choices of $k(l)$. As $q \geq 7$, we can take $[q / 4]+3$ different $k(l)$ values. Then there exist at least three $k=k(l)$ such that both (4) and $k \leq 3 q / 4$ hold. Thus we can choose $k=k(l)$ so that $C, D \in S_{1}$. (Here we used the fact $(n / q, q)=1$.) Now consider the case $a \in[36, n / 4)$, and take the integer $i$ with $a \in\left[n / 2^{i+2}, n / 2^{i+1}\right)$. Similarly to Lemma 7 , we see that $a A, a B, a C$ and $a D$ cannot lie in $S_{1}$ simultaneously. Finally, we consider the case $a \leq 35$. If $a \in[7,35]$ then $a^{2} \in[36, n / 4)$. If $a=5$ then $5^{3} S_{1}=S_{1}$ and $5^{3} \in[36, n / 4)$. This completes the proof.

Proof of the Theorem. By using the Proposition, very suitable for numerical calculations, we can easily check the assertion of the Theorem for $n \leq 70^{2}$. Combining Lemmas $4-7$, if $n$ is a counterexample, we see that $n=12 m$ and $(m, 6)=1$ with a square free integer $m$. Now in view of Lemma 8, the greatest prime factor of $m$ is 5, which yields case 4 of the Theorem. This completes the proof.

Acknowledgments. I am very grateful to Yoshio Mimura and Akihiro Munemasa who showed me this problem and the relevant computational phenomena. Thanks are also due to Mieko Yamada who kindly permitted me to include her proof of Lemma 3 in this article. I would also like to express my best gratitude to my colleague, Teruo Takeuchi, with whom I discussed the subject. The essential part of the proof of Lemma 4 is due to him.

\section{References}

[1] L. D. Baumert, W. H. Mills and R. L. Ward, Uniform cyclotomy, J. Number Theory 14 (1982), 67-82.

[2] B. C. Berndt and R. J. Evans, Sums of Gauss, Jacobi, Jacobsthal, ibid. 11 (1979), 349-398. 
[3] B. C. Berndt and R. J. Evans Sums of Gauss, Eisenstein, Jacobi, Jacobsthal, and Brewer, Illinois J. Math. 23 (1979), 374-437.

[4] - - - The determination of Gauss sums, Bull. Amer. Math. Soc. (N.S.) 5 (1981), $107-129$.

[5] -, 一, Corrigendum to "The determination of Gauss sums", ibid. 7 (1982), 441.

[6] S. Chowla, On Gaussian sums, Proc. Nat. Acad. Sci. U.S.A. 48 (1962), 1127-1128.

[7] R. J. Evans, Generalization of a theorem of Chowla on Gaussian sums, Houston J. Math. 3 (1977), 343-349.

[8] - Resolution of sign ambiguities in Jacobi and Jacobsthal sums, Pacific J. Math. 81 (1979), 71-80.

[9] -, Pure Gauss sums over finite fields, Mathematika 28 (1981), 239-248.

[10] T. Ito, H. Ishibashi, A. Munemasa and M. Yamada, The Terwilliger algebra of cyclotomic schemes and rationality of Jacobi Sums, in: Abstracts of the Conference on Algebraic Combinatorics, Fukuoka, 1993, 43-44.

[11] D. S. Kubert and S. Lang, Independence of modular units on Tate curves, Math. Ann. 240 (1979), 191-201.

[12] S. Lang, Cyclotomic Fields, I and II, Graduate Texts in Math. 121, Springer, 1990.

[13] L. Mordell, On a cyclotomic resolvent, Arch. Math. (Basel) 13 (1962), 486-487.

Faculty of Science

Niigata University

Niigata, 950-21, Japan

E-mail: akiyama@geb.ge.niigata-u.ac.jp 